\title{
Comparative Studies on Various Room-Temperature Vulcanizing-Type Silicone Rubbers as Ion-Selective Electrode Matrices
}

\author{
Kyung Lae RHo*, Sang Hyun HaN*, Hakhyun NAM*, Geun Sig ChA*, \\ Kyoung Il JounG**, Yong Woo KIM** and Ki-Jung PAENG** \\ * Department of Chemistry, Kwangwoon University, \\ 447-1 Wolgye-Dong, Nowon-Ku, Seoul 139-701, Korea \\ ** Department of Chemistry, Yonsei University, Wonju, 220-710, Korea
}

\begin{abstract}
In order to investigate the performance of different types of silicone rubber (SR) materials as matrices for ion-selective membrane electrodes, various cation-selective membranes are formulated from each of four different room temperature vulcanizing (RTV) SRs with addition of plasticizer. Four different neutral carrier-type cation ionophores $\left(\mathrm{K}^{+}, \mathrm{Ca}^{2+}\right.$, and two $\mathrm{Na}^{+}$) were included in this study. These SR-based ion-selective membranes were compared with the corresponding PVC-based membranes in both conventional electrode body and coated wire-type electrode formats. All of the onecomponent RTV SRs except for the oxime-evolving type, were shown to exhibit similar potentiometric response characteristics for a given ionophore system. It was also shown, however, that their performance is greatly dependent on the type of the neutral carrier incorporated.
\end{abstract}

Keywords Ion-selective electrode, room-temperature vulcanizing, silicone rubber, membrane, matrix

Ion-selective electrodes (ISEs) based on plasticized poly(vinyl chloride) (PVC) membranes doped with neutral carriers are now routinely used for the determination of electrolytes in physiological fluids. ${ }^{1-3}$ However, the use of PVC-based membranes in allsolid-state-type electrodes [e.g., coated-wire electrodes (CWEs) and ion-selective field-effect transistors (ISFETs)] results in limited success because of their inherently weak adhesion to most solid surfaces.

In recent years, one-component room temperature vulcanizing (RTV)-type silicone rubber (SR) materials have attracted attention as alternatives to PVC for formulating ion-selective membranes, primarily in an attempt to take advantage of their excellent adhesion to most solid surfaces, in particular to the surface of the base solid-state electrodes..$^{4-10}$ Typically, these materials can be dissolved or dispersed in several organic solvents including tetrahydrofuran, and are easily cured at room temperature by atmospheric moisture activation, which causes a condensation of silicon prepolymers to a high molecular-weight rubber with alcohol ${ }^{4-7}$, acetic acid ${ }^{8-10}$, oxime $^{4}$ and acetone ${ }^{4}$ as a byproduct. Most of these SRs were tested previously as base membranes for ISEs. Kimura et al. ${ }^{4}$ examined alcohol-, acetone- and oximeevolving type SRs concerning their usefulness as membrane matrices in fabricating the calix[4]arene-based $\mathrm{Na}^{+}$-ISFETs. Although all of these three SR-based membranes were reported to show a similar performance, the oxime-evolving type was selected due to its wide use and quick hardening property. It is noted that an alcoholevolving type was employed in a similar experiment later. $^{5}$ The aforementioned experiments by Kimura et al ${ }^{4,5}$ employed only one type of ionophore (i.e., a calix[4]arene derivative) in SR-matrix membranes, without the use of a plasticizer, for the development of $\mathrm{Na}^{+}$-ISFETs. However, the plasticized SR membranes, when optimized, were shown to exhibit enhanced performance in terms of potentiometric response and membrane resistance. 6,7 It was also demonstrated that the addition of a plasticizer to the SR matrix does not significantly deteriorate its adhesive property.

The purpose of this work was to investigate the performance of several different types of SR materials (see Table 1) as matrices for ion-selective membrane electrodes. For this, various cation-selective membranes were formulated from each of four different RTV SRs with addition of a plasticizer. Four different neutral carrier-type cation ionophores $\left(\mathrm{K}^{+}, \mathrm{Ca}^{2+}\right.$, and two $\left.\mathrm{Na}^{+}\right)$ available from commercial sources were included in the present work. These SR-based ion-selective membranes were compared with the corresponding conventional PVC-based membranes. In addition, CWEtype sensors were prepared with the selected membrane formulations, and their electrochemical properties examined. From these results, the relative advantages and disadvantages of each membrane system are discussed. 
Table 1 Classification of silicone rubbers as evolving materials

\begin{tabular}{lcc}
\hline \multirow{2}{*}{ Classification } & \multicolumn{2}{c}{ Matrix type } \\
\cline { 2 - 3 } & Flowable & Nonflowable \\
\hline Alcohol-evolving type & 3140 RTV & 3145 RTV \\
Acid-evolving type & 734 RTV & - \\
Oxime-evolving type & 210 RTV & - \\
\hline
\end{tabular}

\section{Experimental}

\section{Reagents}

Poly(vinyl chloride) (PVC), bis(2-ethylhexyl) sebacate (DOS), bis(2-ethylhexyl) adipate (DOA), potassium tetrakis(4-chlorophenyl)borate (KTpClPB), 4- $t$-butylcalix[4]arene-tetraacetic acid tetraethyl ester (Calix[4]arene), $N, N, N^{\prime}, N^{\prime}$-tetracyclohexyl-1,2-phenylenedioxydiacetamide (ETH 2120), and $N, N, N^{\prime}, N^{\prime}$-tetracyclohexyl3-oxapentanediamide (ETH 129) were purchased from Fluka (Buchs, Switzerland), while valinomycin was purchased from Sigma (St. Louis, MO, USA). All of the one-component silicone rubbers $(3140,3145,734$ and 210 RTVs) were products of Dow Corning (Midland, MI, USA), and are classified according to the evolving byproduct during their hardening in Table 1. All other chemicals used were of analytical-reagent grade. Standard solutions and buffers were prepared with deionized water.

\section{Preparation of polymer membranes}

PVC or SR matrix membranes were prepared according to a previously reported method. ${ }^{10-12}$ The composition of the PVC-based cation-selective membrane was typically $1.0 \mathrm{wt} \%$ ionophore, $33 \mathrm{wt} \%$ PVC and 66wt \% plasticizer with a total weight of $200 \mathrm{mg}$ for a circular membrane (22-mm i.d.). SR membranes were prepared by dissolving appropriate amounts of RTVs, ionophore, plasticizer and lipophilic additive (if necessary) in THF and by casting the mixture in a glass ring (22-mm i.d.) placed on a flat Teflon plate. Table 2 summarizes the compositions of the cation-selective polymer membranes examined in this study. PVC membrane cocktails were dried for at least $24 \mathrm{~h}$, while those based on SRs were dried for 3-5d at room temperature. The thickness of these membranes was approximately $100 \mu \mathrm{m}$.

\section{Evaluating potentiometric response and selectivity}

The potentiometric behaviors of cation-selective membranes were evaluated in the conventional ISE configuration, as described elsewhere. ${ }^{11,12}$ Small disks (5.5 $\mathrm{mm}$ ) were punched from cast films and mounted in Phillips electrode bodies (IS-561; Glasbläserei Möller, Zürich, Switzerland). The internal filling solution was varied depending on the ISE membrane system: $0.1 \mathrm{M}$ $\mathrm{KCl}$ for $\mathrm{K}^{+}, 0.1 \mathrm{M} \mathrm{NaCl}$ for $\mathrm{Na}^{+}$, and $0.1 \mathrm{M} \mathrm{CaCl} 2$ for $\mathrm{Ca}^{2+}$-selective electrodes. Coated-wire electrodes (CWEs) were prepared with membrane formulations selected from Table 2. A silver wire was dipped in a membrane cocktail 10 times and dried at room temperature. An Orion sleeve-type double junction Ag/ $\mathrm{AgCl}$ electrode (Model 90-02) was used as the external reference. The potential differences between the ISEs and the reference electrode were measured using an IBM AT-type computer equipped with a custom highimpedance input $\left(10^{12} \mathrm{ohm}\right) 16$-channel analog-to-digital converter.

The potentiometric behaviors of all electrodes were determined in $0.05 \mathrm{M}$ Tris- $\mathrm{HCl}$ buffer ( $\mathrm{pH} \mathrm{7.2).} \mathrm{The}$ selectivity coefficients were determined from the response potentials in corresponding cation solutions with respect to those of interfering cations $(0.1 \mathrm{M})$ by the separation-matched potential method. ${ }^{13}$ The detection limits of the membrane electrodes were obtained from the calibration curves, as described previously. ${ }^{14}$ The relative bulk membrane electrical resistance was evaluated according to a method described elsewhere. ${ }^{7,15}$

Table 2 Membrane compositions ${ }^{a}$

\begin{tabular}{|c|c|c|c|c|c|c|c|c|c|}
\hline & \multicolumn{2}{|c|}{ Matrix } & \multicolumn{4}{|c|}{ Ionophore } & \multicolumn{2}{|c|}{ Plasticizer. } & \multirow{2}{*}{$\begin{array}{c}\begin{array}{c}\text { Lipophilic }^{\mathrm{b}} \\
\text { additive }\end{array} \\
\text { KTpClPB }\end{array}$} \\
\hline & PVC & SR & Calix[4]arene & ETH 2120 & Valinomycin & ETH 129 & DOS & DOA & \\
\hline \multirow{4}{*}{$\begin{array}{l}\mathrm{Na}^{+} \text {-Selective } \\
\text { membrane }\end{array}$} & 33.0 & - & 1.0 & - & - & - & 66.0 & - & - \\
\hline & - & 87.0 & 1.0 & - & - & - & 12.0 & - & - \\
\hline & 33.0 & - & - & 1.0 & - & - & - & 66.0 & - \\
\hline & - & 87.0 & - & 1.0 & - & - & - & 12.0 & - \\
\hline \multirow{2}{*}{$\begin{array}{c}\mathrm{K}^{+}-\text {Selective } \\
\text { membrane }\end{array}$} & 33.0 & - & - & - & 1.0 & - & 66.0 & - & - \\
\hline & - & 87.0 & - & - & 1.0 & - & 12.0 & - & - \\
\hline \multirow{2}{*}{$\begin{array}{l}\mathrm{Ca}^{2+}-\text { Selective } \\
\text { membrane }\end{array}$} & 33.0 & - & - & - & - & 1.0 & - & 66.0 & 46.4 \\
\hline & - & 87.0 & - & - & - & 1.0 & - & 12.0 & - \\
\hline
\end{tabular}

a. In $\mathrm{wt} \%$. b. In $\mathrm{mol} \%$ relative to the ionophore. 
Table 3 Electrochemical properties of sodium-selective membrane electrodes doped with Calix[4]arene

\begin{tabular}{|c|c|c|c|c|c|c|c|}
\hline & \multirow[t]{2}{*}{ Membrane type } & \multirow{2}{*}{$\begin{array}{c}\text { Slope }^{\mathrm{a}} \\
(\mathrm{mV} / \text { decade })\end{array}$} & \multirow{2}{*}{$\begin{array}{l}\text { Detection limit, } \\
\qquad \log a_{\mathrm{Na}^{+}}\end{array}$} & \multicolumn{3}{|c|}{$\begin{array}{l}\text { Selectivity coefficient }{ }^{\mathrm{b}}, \\
\log k_{\mathrm{Na}^{+}, \mathrm{j}}^{\mathrm{pot}^{+}} \\
\end{array}$} & \multirow[t]{2}{*}{ Resistance/M $\Omega$} \\
\hline & & & & $\mathbf{K}^{+}$ & $\mathrm{Ca}^{2+}$ & $\mathrm{Mg}^{2+}$ & \\
\hline $\mathbf{A}$ & PVC & 57.9 & -5.71 & -2.77 & -1.53 & -4.35 & $91-93$ \\
\hline B & 3140 RTV & 58.3 & -5.77 & -2.74 & -1.55 & -4.43 & $1230-1380$ \\
\hline C & 3145 RTV & 57.8 & -5.75 & -2.75 & -1.58 & -4.44 & $1260-1610$ \\
\hline D & 734 RTV & 57.9 & -5.71 & -2.81 & -1.61 & -4.53 & $506-508$ \\
\hline $\mathrm{E}$ & 210 RTV & - & - & - & - & - & - \\
\hline
\end{tabular}

a. Range: $10^{-4}-10^{-1}$ M. b. Separation-matched potential method.

\section{Results and Discussion}

As reported previously, ${ }^{6,7}$, the electrochemical behavior of a 3140 RTV-based ISE membrane can be improved by incorporating a plasticizer (about $10-35 \mathrm{wt} \%$ ) into the membrane phase. On the basis of these results, preliminary experiments were carried out first to determine the type and amount of plasticizer of the 3140 RTV-based membranes for each ionophore system examined in the present work. These compositions were then applied to other SR-based membrane systems without further optimization. On the other hand, the composition of PVC-based membranes were those recommended based on previous studies or by Fluka ${ }^{16}$, all being the typical ISE membrane compositions.

\section{Evaluation of $\mathrm{Na}^{+}$-selective membranes}

As mentioned in the Introduction section, Kimura et al. had already tested several different types of onecomponent RTVs as ion-selective membrane matrices. ${ }^{4}$ In their experiments, calix[4]arene derivatives were employed as neutral carriers, without using a plasticizer, to prepare membrane casting solutions for fabricating $\mathrm{Na}^{+}$-ISFETs. In the present work, however, we prepared $\mathrm{Na}^{+}$-selective membranes by incorporating a calix[4]arene derivative (i.e., denoted as Calix[4]arene), this time to plasticized SR matrices (see Table 2 for the membrane compositions).

As can be seen in Table 3 , the potentiometric performances of Calix[4]arene-based $\mathrm{Na}^{+}$-ISEs with SR matrices except for the oxime-evolving $210 \mathrm{RTV}$, were very similar to that of the corresponding PVC matrix system. It should be mentioned that $\mathrm{Na}^{+}$-ISFETs employing SR membranes doped with the same calix[4]arene derivative, but without a plasticizer, did not perform well, as previously reported. ${ }^{4,5}$ This poor response was reported to be derived mainly due to the low solubility of the calixarene ionophore in the SR matrices. ${ }^{4}$ It is believed that a plasticizer added to the SR matrix helps to increase the solubility of ionophores doped in the membrane, resulting in a much improved potentiometric performance, as demonstrated in Table 3 . However, the membrane prepared with 210 RTV did not show any significant response to the cations tested,

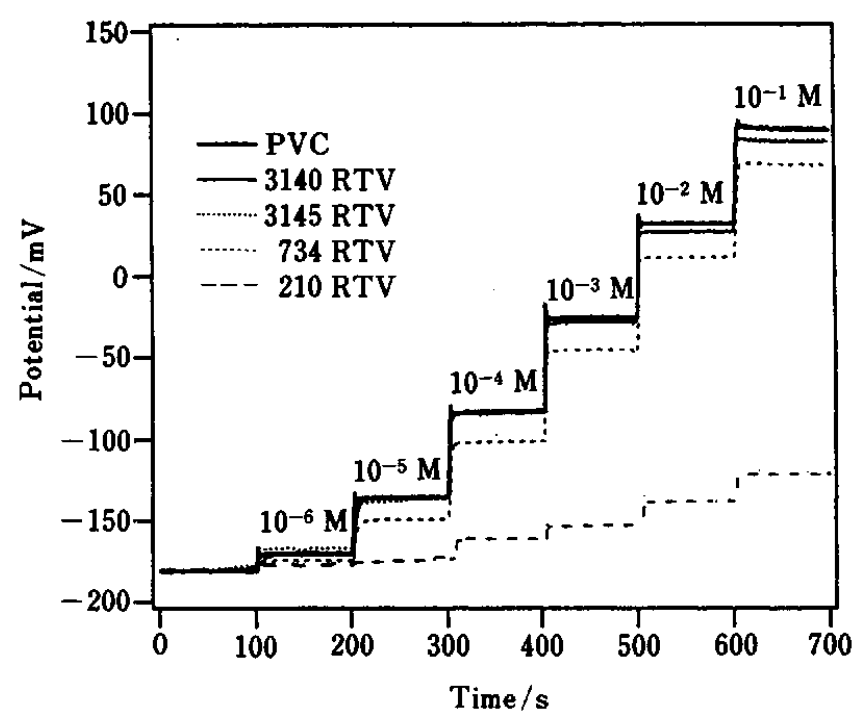

Fig. 1 Response characteristics of PVC and silicone rubber membrane-based $\mathrm{Na}^{+}-\mathrm{CWEs}$ doped with Calix[4]arene.

despite its acceptable physical properties as an electrode membrane. As is typical with SR matrices, all of the $\mathrm{Na}^{+}$-selective SR membranes possessed relatively high electrical resistances, much higher than that of the PVC membrane, as can be seen in Table 3. It can also be seen that the alcohol-evolving type SR (3140 RTV and 3145 RTV) membranes exhibit higher electrical resistances than acid-evolving SR (734 RTV) with Calix[4]arene doped as the ionophore.

Figure 1 illustrates the dynamic-response properties of $\mathrm{Na}^{+}$-CWEs prepared with the membrane formulations used for the Calix[4]arene-based ISEs. Similar to the ISE experiments, SR-based $\mathrm{Na}^{+}$-CWEs exhibited electrochemical performance comparable to that of PVC, except for the 210 SR-based CWE. It should be mentioned that the detection limit of the 734 RTV-based membrane and the selectivity of 3145 RTV (with respect to $\mathrm{K}^{+}$) degrade somewhat in CWE experiments.

As shown, the performance of Calix[4]arene-based SR-matrix membranes can be significantly influenced by the type of SR matrices employed. In order to further 
Table 4 Electrochemical properties of sodium-selective membrane electrodes doped with ETH 2120

\begin{tabular}{|c|c|c|c|c|c|c|c|}
\hline & \multirow[t]{2}{*}{ Membrane type } & \multirow{2}{*}{$\begin{array}{c}\text { Slope }^{\mathrm{a}} \\
\text { (mV/decade) }\end{array}$} & \multirow{2}{*}{$\begin{array}{l}\text { Detection limit, } \\
\qquad \log a_{\mathrm{Na}^{+}}\end{array}$} & \multicolumn{3}{|c|}{$\begin{array}{l}\text { Selectivity coefficient } \\
\qquad \log k_{\mathrm{Na}^{+}, \mathrm{j}}^{\text {pot }}\end{array}$} & \multirow[t]{2}{*}{ Resistance/M $\Omega$} \\
\hline & & & & $\mathbf{K}^{+}$ & $\mathrm{Ca}^{2+}$ & $\mathrm{Mg}^{2+}$ & \\
\hline A & PVC & 55.4 & -4.74 & -1.53 & -2.08 & -3.99 & $42-43$ \\
\hline B & 3140 RTV & 55.2 & -5.12 & -1.57 & -1.84 & -3.91 & $220-240$ \\
\hline C & 3145 RTV & 56.4 & -4.59 & -1.43 & -1.95 & -3.67 & $290-400$ \\
\hline D & 734 RTV & 52.9 & -4.88 & -1.50 & -1.12 & -3.81 & $370-390$ \\
\hline $\mathrm{E}$ & 210 RTV & - & - & - & - & - & - \\
\hline
\end{tabular}

a. Range: $10^{-4}-10^{-1}$ M. b. Separation-matched potential method.

examine the plasticized SR-matrix systems, an additional $\mathrm{Na}^{+}$ionophore (ETH 2120) was utilized (see Table 2 for membrane compositions); the results are summarized in Table 4. Again, the oxime-evolving 210 RTV did not yield membranes with measurable potentiometric signals. The response properties of other SR-matrix membranes were similar to those of the PVC-based membrane. Unlike the Calix[4]arene-based system, the electrical resistance was not very different between the alcohol-evolving and acid-evolving types for the ETH 2120 ionophore system. This may be explained by an enhanced solubility of ETH 2120 in the alcohol-evolving type SR. Overall, the potentiometric performance of the ETH 2120-based $\mathrm{Na}^{+}$-ISE membranes were far inferior to that of the Calix[4]arenebased ones (i.e., compare slopes, detection limits and selectivities listed in Tables 3 and 4). With both ionophores, however, the response times of the SR-based $\mathrm{Na}^{+}$-ISEs were very fast $(1-3 \mathrm{~s})$, relatively stable for a long period of time (3-4h), and were virtually the same as those of the corresponding PVC-based membranes.

In the ETH 2120-based $\mathrm{Na}^{+}-\mathrm{CWE}$ experiments (Fig. 2), all of the membranes, except for the 210 RTVbased one, resulted in sensors with relatively good response properties. It can be seen that the 3140 and 3145 RTV-based CWEs showed even better detection limits than did the PVC-based CWE. However, the overall response properties of ETH 2120-based electrodes, while having lower electrical resistances, were inferior to those of Calix[4]arene-based electrodes. This observation may support the fact that the bulk membrane resistance is not of major importance in determining the potentiometric performance of the ISE membrane. High-resistance membranes can be analytically useful if the instrument has an input impedance greater than $10^{4}$ times of the membrane resistance. ${ }^{17}$

\section{Evaluation of $K^{+}$-selective membranes}

Valinomycin is certainly one of the most successful ionophores used in polymeric ion-selective membrane electrodes. PVC-matrix membranes doped with valinomycin have been successfully used for the determination of $\mathrm{K}^{+}$concentrations in a variety of samples. However, it has been known that such PVC-

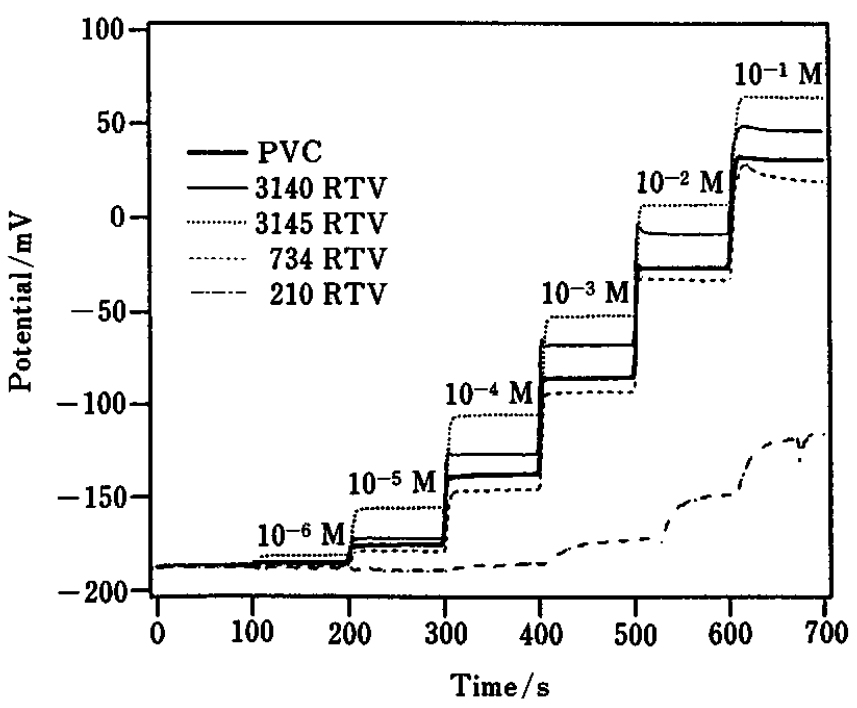

Fig. 2 Response characteristics of PVC and silicone rubber membrane-based $\mathrm{Na}^{+}$-CWEs doped with ETH 2120 .

matrix membranes suffer from lipophilic anion interference that may be in undiluted urine, whole blood or serum samples. This shortcoming was reported to be overcome by employing Siloprene, a two-component $\mathrm{SR}$, as the matrix for valinomycin-based $\mathrm{K}^{+}$-selective membranes. ${ }^{18}$ Such membranes are now in routine use for the bed-side monitoring of undiluted urine. Unlike Siloprene, all of the SR materials examined in the present study are one-component systems and adhered tightly to most solid surfaces. In addition, membranes prepared with such RTV materials possess improved biocompatibility and showed less interference from lipophilic anions. ${ }^{19,20}$

Table 5 summarizes the electrochemical properties of PVC and one-component SR membranes incorporating valinomycin (see Table 2 for each membrane composition). Again, the alcohol- (3140 and 3145 RTVs) and acid-evolving type (734 RTV) SR membranes exhibited a response performance comparable to that of PVC, in terms of the slope, detection limit and selectivity. It should be noted, however, that, this time, considerable response signals were observed even with the oxime- 
Table 5 Electrochemical properties of potassium-selective membrane electrodes doped with valinomycin

\begin{tabular}{|c|c|c|c|c|c|c|c|}
\hline & \multirow[t]{2}{*}{ Membrane type } & \multirow{2}{*}{$\begin{array}{c}\text { Slope }^{a} \\
\text { (mV/decade) }\end{array}$} & \multirow{2}{*}{$\begin{array}{l}\text { Detection limit, } \\
\qquad \log a_{\mathrm{K}^{+}}\end{array}$} & \multicolumn{3}{|c|}{$\begin{array}{l}\text { Selectivity coefficient }{ }^{\mathrm{b}}, \\
\qquad \log k_{\mathbf{K}^{+}, \mathrm{j}}^{\text {pot }}\end{array}$} & \multirow[t]{2}{*}{ Resistance/M $\Omega$} \\
\hline & & & & $\mathrm{Na}^{+}$ & $\mathrm{Ca}^{2+}$ & $\mathbf{M g}^{2+}$ & \\
\hline A & PVC & 56.6 & -6.49 & -4.43 & -4.75 & -4.96 & $51-53$ \\
\hline B & 3140 RTV & 57.4 & -6.50 & -4.43 & -4.70 & -4.94 & $140-176$ \\
\hline $\mathrm{C}$ & 3145 RTV & 57.2 & -6.50 & -4.44 & -4.72 & -4.98 & $77-80$ \\
\hline D & 734 RTV & 57.2 & -6.38 & -4.36 & -4.70 & -4.86 & $110-170$ \\
\hline $\mathrm{E}$ & 210 RTV & 43.1 & -4.74 & -3.46 & -3.17 & -4.00 & - \\
\hline
\end{tabular}

a. Range: $10^{-5}-10^{-1} \mathrm{M}$. b. Separation-matched potential method.

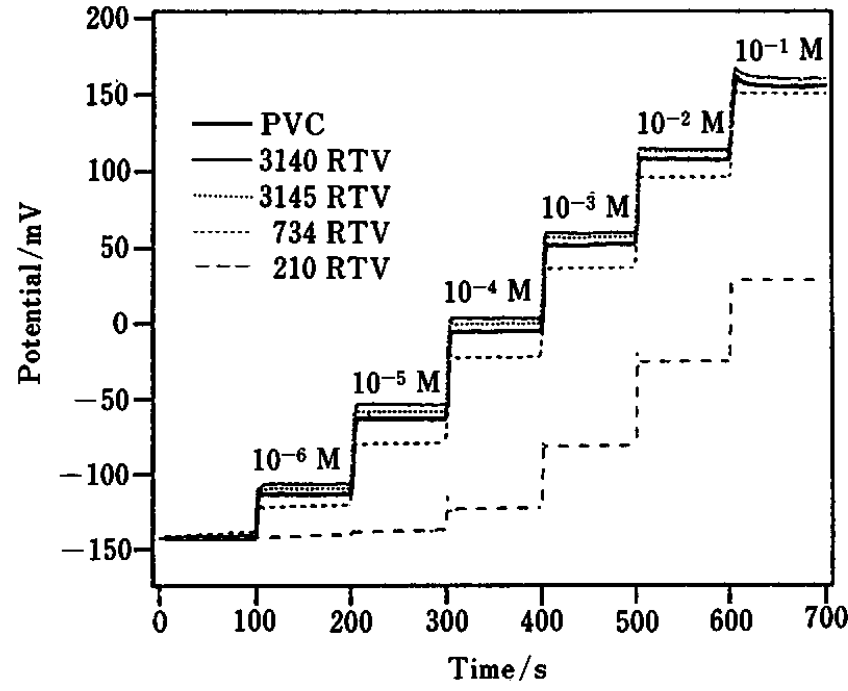

Fig. 3 Response characteristics of $\mathrm{PVC}$ and silicone rubber membrane-based $\mathrm{K}^{+}-\mathrm{CWEs}$ doped with valinomycin.

evolving type (210 RTV) membrane: both Calix[4]areneand ETH 2120-doped 210 RTV membranes exhibited negligible ion response. Furthermore, the electrical resistances of silicone rubber membranes were much lower when doped with valinomycin than with other ionophores including Calix[4]arene and ETH 2120. The valinomycin-based membrane system appears to be less influenced by the type of matrix employed, and, in fact, most plasticizer-free SR membranes doped with valinomycin are reported to function satisfactorily. These observations may indicate that valinomycin is mixed homogeneously and diffuses relatively freely through the matrix in the SR membranes, regardless of the presence of plasticizer. Since a sufficiently high mobility of the neutral carrier molecule is a prerequisite for the functioning of the ISE, well dissolving ionophore in SR matrices represents the low resistance and enhanced electrochemical performance.

Figure 3 shows the potentiometric responses of valinomycin-based $\mathrm{K}^{+}$-CWEs to added potassium. All of the CWEs include 210 RTV-based one performed in a fashion similar to the corresponding ISEs.

\section{Evaluation of $\mathrm{Ca}^{2+}$-selective membranes}

The observed limitations of most SR-based sensors, particularly those prepared without a plasticizer, are their high resistances and slower response times to active steps. These drawbacks were interpreted by assuming the existence of a high-resistance film on the surface of the membrane and the forming crystalline by the ionophore in the membrane phase. ${ }^{21,22}$ In some cases, these problems can be overcome by the addition of a plasticizer (i.e., to reduce the membrane resistance and increase the solubility of active membrane components in the SR membrane phase). For instance, the performance of the $3140 \mathrm{RTV}$-type SR-based $\mathrm{Ca}^{2+}$-ISEs doped with ETH 1001 or ETH 129 can be significantly improved by the addition of a plasticizer. ${ }^{7}$ Carefully optimized SR membranes were shown to exhibit a potentiometric performance even better than that of PVC-matrix membranes.

In this work, we prepared plasticized SR-based $\mathrm{Ca}^{2+}$ ISE membranes employing ETH 129 as the ionophore (see Table 2 for each membrane composition). As can be seen in Table 6, ETH 129-doped plasticized SRmatrix membranes exhibited response behaviors different from those observed with other ionophorebased SR membrane systems: slopes were overNernstian, the selectivities degraded significantly, and resistances were very high (out of range with the method employed in this work). The observed over-Nernstian behavior suggests that the charged associates of the ionophore and monovalent calcium complex ions (e.g., $\left.\left[\mathrm{Ca}(\mathrm{ETH} 129)_{n} \mathrm{Cl}\right]^{+}\right)$are part of the permeating species in those membranes. ${ }^{7,23}$ Membranes with such superNernstian behavior should not be a problem by themselves, particularly from an analytical standpoint, but they usually display poor reproducibility. This abnormal response property of plasticized $\mathrm{Ca}^{2+}$-selective SR membranes was reported to be corrected by incorporating a lipophilic salt, such as tetradodecylammonium tetrakis ( $p$-chlorophenyl)borate. ${ }^{7}$ We also prepared $\mathrm{Ca}^{2+}-\mathrm{CWEs}$ and observed response slopes and selectivities similar to the corresponding ISEs (not shown). It should be mentioned that the enhanced lifetimes of SR-based CWE-type sensors, compared to those of PVC-based CWEs, were demonstrated in our previous work. ${ }^{6,7}$ 
Table 6 Electrochemical properties of calcium-selective membrane electrodes doped with ETH 129

\begin{tabular}{|c|c|c|c|c|c|c|c|}
\hline & \multirow[t]{2}{*}{ Membrane type } & \multirow{2}{*}{$\begin{array}{c}\text { Slope }^{\mathrm{a}} \\
\text { (mV/decade) }\end{array}$} & \multirow{2}{*}{$\begin{array}{l}\text { Detection limit, } \\
\qquad \log a_{\mathrm{Ca}^{2+}}\end{array}$} & \multicolumn{3}{|c|}{$\begin{array}{c}\text { Selectivity coefficient } \\
\log k_{\mathrm{Ca}^{2+}}^{\text {pot }}\end{array}$} & \multirow[t]{2}{*}{ Resistance/M $\Omega$} \\
\hline & & & & $\mathbf{K}^{+}$ & $\mathrm{Na}^{+}$ & $\mathrm{Mg}^{2+}$ & \\
\hline A & PVC & 28.1 & -5.79 & -4.46 & -4.71 & -4.72 & $9-16$ \\
\hline B & 3140 RTV & 48.8 & -5.61 & -4.14 & -3.32 & -4.56 & - \\
\hline $\mathrm{C}$ & 3145 RTV & 44.9 & -5.41 & -4.23 & -3.64 & -4.53 & - \\
\hline D & 734 RTV & 42.1 & -5.70 & -4.04 & -3.55 & -4.46 & - \\
\hline$E$ & $210 \mathrm{RTV}$ & - & - & - & - & - & - \\
\hline
\end{tabular}

a. Range: $10^{-4}-10^{-1} \mathrm{M}$. b. Separation-matched potential method. c. Resistances of membrane $\mathrm{B}-\mathrm{E}$ were out of range with the method employed.

It has been our experience that blank SR-matrix membranes (i.e., ionophore-free) incorporating even a lipophilic ionic additive or a plasticizer exhibit a significant response to $\mathrm{pH}$ changes, particularly in the range of acidic $\mathrm{pH}^{24}$ This was assumed to be due to the presence of ionic sites (e.g., silanol groups or ionizable impurities) within the RTVSR membrane phase. However, the $\mathrm{pH}$ response of the SR membranes usually disappears when doped with neutral carrier-type cation ionophores. It appears that the neutral carriermediated selectivity overrides the intrinsic $\mathrm{pH}$ response of the silanol-terminated SR-matrix membranes. This was demonstrated again in the present work: all of the membranes doped with neutral carriers (i.e., ETH 129, ETH 2120, Calix[4]arene, valinomycin) indeed exhibited a negligible $\mathrm{pH}$ response (less than $1 \mathrm{mV}$ per unit $\mathrm{pH}$ change), except for the 210 RTV-based SR membranes which showed a relatively large $\mathrm{pH}$ response in the range of acidic $\mathrm{pH}(15-30 \mathrm{mV}$ per unit $\mathrm{pH}$ change).

In summary, several different types of one-component RTV SRs were compared as alternative matrices for ionselective membranes. The electrochemical properties of the SR membranes were shown to be greatly dependent upon the types of both the SR matrix and the ionophore system employed. In general, the alcohol- and acidevolving type SR-membranes, when plasticized, exhibited response properties comparable to those of PVCmatrix membranes. On the other hand, plasticized oxime-evolving SRs did not yield ISE membranes with useful performances. The response properties of CWEs prepared with SR-based membrane formulations were similar to those of the corresponding ISEs.

This work was supported in part by the Ministry of Education, Korea, through the Basic Science Research Institute Program (BSRI-96-3405), and by Maeji Institute of Academic Research, Yonsei University in the school year 1997. Partial support by Kwangwoon University is also acknowledged.

\section{References}

1. J. D. R. Thomas, Anal. Chim. Acta, 180, 289 (1986).

2. D. M. Pranitis, M. Telting-Diaz and M. E. Meyerhoff, Crit. Rev. Anal. Chem., 23, 163 (1992).
3. S. Nomura, Analyst [London], 120, 503 (1995).

4. K. Kimura, T. Matsuba, Y. Tsujimura and $M$. Yokoyama, Anal. Chem., 64, 2508 (1992).

5. Y. Tsujimura, M. Yokoyama and K. Kimura, Anal. Chem., 67, 2401 (1995).

6. J. H. Shin, D. S. Sakong, H. Nam and G. S. Cha, Anal. Chem., 68, 221 (1996).

7. B. K. Oh, C. Y. Kim, H. J. Lee, K. L. Rho, G. S. Cha and H. Nam, Anal. Chem., 68, 503 (1996).

8. M. Knoll, K. Cammann, C. Dumschat, C. Sundermeier and J. Eshold, Sens. Actuators B, 51, 18 (1994).

9. G. Högg, O. Lutze and K. Cammann, Anal. Chim. Acta, 335, 103 (1996).

10. G. S. Cha, D. Liu, M. E. Meyerhoff, H. C. Cantor, A. R. Midgey, H. D. Goldberg and R. B. Brown, Anal. Chem., 63, 1666 (1991).

11. M. J. Cha, J. H. Shin, B. K. Oh, C. Y. Kim, G. S. Cha, D. S. Shin and B. Kim, Anal. Chim. Acta, 315, 311 (1995).

12. K.-H. Kwon, K.-J. Paeng, D. K. Lee, I. C. Lee, U. S. Hong and G. S. Cha, J. Chromatogr., 688, 350 (1994).

13. Y. Umezawa, K. Umezawa and H. Sato, Pure Appl. Chem., 67, 507 (1995).

14. R. P. Buck and E. Linder, Pure Appl. Chem., 66, 2527 (1994).

15. D. Diamond and F. Regan, Electroanalysis, 2, 113 (1990).

16. Fluka Selectophore Catalogue, Fluka Chemie AG, Buchs, Switzerland, 1996.

17. E. Pungor, Electroanalysis, 8, 348 (1996).

18. P. Anker, H.-B. Jenny, U. Wuthier, R. Asper, D. Ammann and W. Simon, Clin. Chem., 29, 1447 (1983).

19. P. D. van der Wal, M. Skowronska-Ptasinka, A. van den Berg, P. Bergveld, E. J. R. Sudhölter and D. N. Reihouldt, Anal. Chim. Acta, 231, 41 (1990).

20. M. Mascini and G. Marrazza, Anal. Chim. Acta, 231, 125 (1990).

21. E. Linden, Zs. Niegreisz, K. Toch, E. Pungor, T. R. Berube and R. P. Buck, J. Electroanal. Chem. Interfacial Electrochem., 259, 67 (1989).

22. P. D. van der Wal, E. J. R. Sudhölter, B. A. Boukamp, H. J. M. Bouwmeester and D. N. Reihouldt, J. Electroanal. Chem. Interfacial Electrochem., 317, 153 (1991).

23. W. E. Morf, "The Principles of Ion-Selective Electrodes and Membrane Transport", p.264, Elsevier, Amsterdam, 1981.

24. J. H. Shin, H. J. Lee, C. Y. Kim, B. K. Oh, K. L. Rho, H. Nam and G. S. Cha, Anal. Chem., 68, 3166 (1996).

(Received May 26, 1997)

(Accepted August 18, 1997) 